\title{
SLOW RELAXATION OF DILUTED ANTIFERROMAGNETS
}

\author{
U. NOWAK and K.D. USADEL
}

Theoretische Physik and SFB 166, Universität Duisburg, Lotharstraße 1, 4100 Duisburg 1 / FRG

Threedimensional diluted Ising-antiferromagnets cooled in a uniform external magnetic field develop a frozen domain state. The relaxation of the domain state after switching off the external field is investigated in detail by a Monte Carlo simulation. A new interpretation of the results leads to a possible connection to the theory of self-organized criticallity proposed by Bak et.al.

In recent years, there have been several theoretical studies on the behavior of random-field systems. Experiments are often performed on diluted Ising-type antiferromagnets in a uniform external magnetic field (DAFF) which belong to the same universality class as the random-field Ising model (RFIM) (1). Like the RFIM the DAFF develops a metastable domain state if the system is cooled in a field from the paramagnetic phase (2). The domain state relaxes towards long-range order after switching off the field. This relaxation is non-exponential due to pinning of the domain walls at vacancies. Since the domain state carries surplus magnetization and internal energy a slow decay of these remanent quantities can be observed. The remanent magnetization of an experimental realization of the DAFF was found by Kleemann et. al. (3). In an earlier publication we investigated the mechanisms for this relaxation by a monte carlo simulation (4) which showed that processes which are relevant for the relaxation primarily take place in the domain walls confirming ideas published earlier by Nattermann and Vilfan (5). From the results of our simulations we could not distinguish whether the decay of the magnetization follows a power-law or a logarithmic law. Therefore, we increased the time of a Monte Carlo run to up to 100000 Monte Carlo steps per spin (MCS). In analysing our data carefully we observed a structure in the fluctuations of the data leading to a new kind of interpretation of the relaxation which will be reported in this letter.

As in our earlier publication we have performed monte carlo simulations on a threedimensional simple cubic lattice with a size of $61 * 61 * 60$ and a site-dilution of $50 \%$. Only nearest neighbour interaction is considered. We used helical boundary conditions and the heat-bath algorithm. As in our earlier work we prepared the domain state choosing a fixed temperature $T$ and field $B$ and performing 1000 MCS starting from a completely random spin configuration. Within this time the random spin configuration relaxes to a practically frozen domain state serving as the initial configuration for the computation of the remanent magnetization and energy.

Analysing our data we observed that fuctuations of the remanent quantities which made the data fitting am- bigous in the earlier simulations occur in the longer simulations as well but now also on longer time scales. The decaying remanent quantities fluctuate around their mean value in a very broad range of time scales. This is demonstrated in fig. 1 which shows a log-log plot of the remanent magnetization versus time.

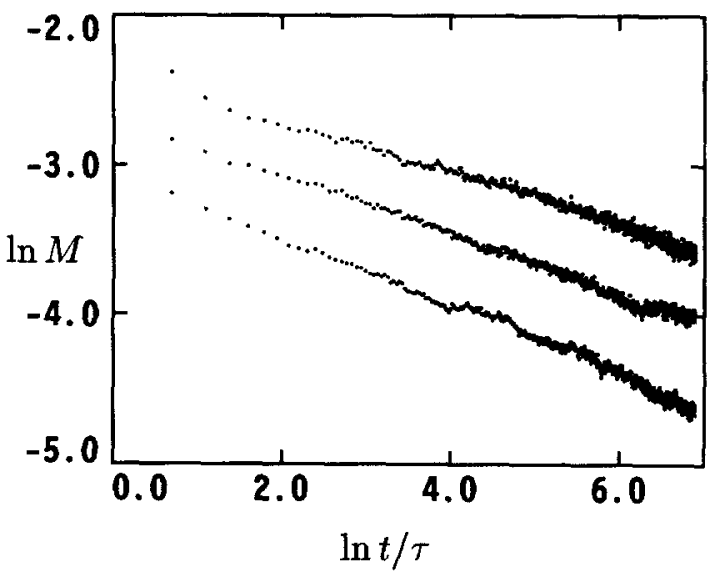

FIGURE 1

The upper curve shows the magnetization of the first 1000 MCS after switching off an external field of $B_{i}=2.0$ at a temperature $T=0.8$. The intermediate curve shows the magnetization of the first 10000 MCS of the same run rescaled in time by a factor of $10, i$. e. every point is an average of 10 consecutive MCS. The lower curve is again rescaled by a factor of 10 and every point is an average of 100 consecutive MCS. Similar behaviour was found for the energy and for different temperatures and fields. From these results we are led to conclude that there are relaxation processes on all time scales leading to a fluctuating magnetization $M(t)$ which has a self similar structure, i. e. it shows the same qualitative behavior on all time scales $\tau$. This lack of a typical time scale in 
our opinion can be explained by a lack of a typical length scale in the system. Above the percolation threshold $p_{c}$ for the vacancies ( $31 \%$ vacancies) there exists a percolating vacancy cluster which is a fractal below a length scale $\xi \sim\left(p-p_{c}\right)^{-\nu}$. Apart from this the domain wall by itself is a fractal as has been shown by Cambier and Nauenberg (7) for the RFIM. Since the relaxation of the domain state takes place in the domain wall or is caused by movements of the domain walls the fractality of the domain wall as well as the fractality of the vacancy clusters result in a lack of typical length scales in the system leading to a wide range of energy barriers with long range temporal correlations. This lack of typical time scales during the relaxation naturally involves a power-law decay for the remanent quantities. Indeed we could confirm the powerlaw as best fitted by averaging over several dilution configurations. We found the exponent of the power-law to be proportional to the temperature only slightly depending on the initial field but different for magnetization and energy.

Within a recent theory of self organized criticallity (SOC) from Bak et. al.(6) the lack of typical time scales leads to a $\omega^{-\alpha}$. law for the power noise spectrum with $\alpha \approx 1$. In order to analyse our simulation data along these lines we have calculated the power spectrum of the noise data $S(\omega)$ :

$$
S(\omega)=\left|\int e^{i \omega t} \Delta M(t) d t\right|^{2}
$$

where $\Delta M(t)$ is the deviation of magnetization data from the best-fitted power-law. A similar analysis was performed for the energy $\Delta E$. Fig. 2 shows the log-log plot of $S(\omega)$ for a temperature of 0.6 and a field $B_{i}$ of 2.0 .

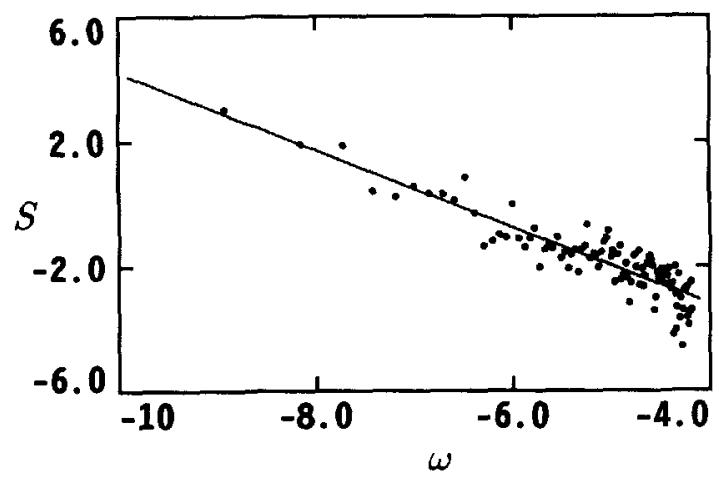

FIGURE 2

The possible values for $\omega$ varied from $\omega_{\min }=\frac{2 \pi}{T}$ to $\omega_{\max }=\frac{\pi}{\Delta t}$ where $T$ is the simulation time (100000 MCS for the long time simulation) and $\Delta t$ is the smallest computed time step (100 MCS). Even though we have averaged the data of $S(\omega)$ over slightly different $\omega$ the data are quite unexact and therefore the resulting exponent is only roughly determined.

In order to get a higher accuracy we have analysed the data for different temperatures and initial fields $B_{i}$ The values of $\alpha$ fluctuate arround $\alpha \approx 1$ in agreement with predictions by SOC theory. No systematical temperature dependence of $\alpha$ is observed and the results for magnetization and energy are practically the same as far as the exponent $\alpha$ is concerned.

The analogy between the theory of SOC from Bak et. al. and our simulations now becomes apparent. A crucial ingredient of the SOC model is an underlying dissipativ transport. This dissipativ transport is realized in our relaxation process by the disappearence of magnetization and internal energy stored in the unsatisfied bonds of the domain wall. However, there is a difference between the dynamics in our situation compared to the usual setup investigated by Bak and others: our system is not an open system in a steady state. However, our system is out of equilibrium and far away from reaching equilibrium for the times investigated and it is open in the sence that there is a dissipativ transport of energy into the heat-bath. Therefore in our opinion the analogy is strong enough to consider the DAFF to be in a SOC state during the relaxation at least for the time scales investigated.

\section{REFERENCES}

(1) S. Fishman and A. Aharony, J.Phys. C12, (1979), $\mathrm{L} 729$

(2) G. S. Grest, C. M. Soukoulis, and K. Levin, Phys.Rev.B 33, (1986), 7659

(3) U. A. Leitão, W. Kleemann, and I. B. Ferreira, Phys.Rev.B 38, (1988), 4765

P. Pollak, W. Kleemann, D. P. Belanger, Phys.Rev.B 38, (1988), 4773

(4) U. Nowak and K. D. Usadel, Phys.Rev.B 39, (1989), 2516

(5) T. Nattermann and I. Vilfan, Phys.Rev.Lett. 61, (1988), 223

(6) J. A. Cambier and M. Nauenberg, Phys.Rev.B 34, (1986), 7998

(7) P. Bak, C. Tang, and K. Wiesenfeld, Phys.Rev.Lett. 59, (1987), 381

C. Tang and P. Bak, Phys.Rev.Lett. 60, (1988), 2347

T. Hwa and M. Kardar, Phys.Rev.Lett. 62, (1989), 1813

D. Dahr and R. Ramaswamy, Phys.Rev.Lett. 63, (1989), 1659 and references therein 